\title{
Potential Tornado Warning Improvement Resulting from Utilization of the TDS in the Warning Decision Process
}

\author{
MATTHEW S. VAN DEN BROEKE \\ University of Nebraska-Lincoln, Lincoln, $N E$
}

(Manuscript received 9 September 2016; review completed 15 May 2017)

\begin{abstract}
Polarimetric tornadic debris signatures (TDSs) provide a means of confirming that a strong wind field has lofted debris to the altitude of the radar beam. They can increase confidence in an ongoing tornado, which may be noted in tornado warning text to increase the sense of urgency. They may also serve as indicators of weak tornadoes that may otherwise go unwarned. Polarimetric data have been available since 2012 on a large portion of the U.S. Weather Surveillance Radar-1988 Doppler (WSR-88D) network and since May 2013 for the entire network. It is anticipated that use of the polarimetric TDS in the tornado warning process should evolve through time. Thus, this paper presents an overview of how the TDS was used in the warning process during the 16 months when widespread polarimetric data were first becoming available (February 2012-May 2013). During this period, $22.5 \%$ of tornado warning texts for TDS-producing events mentioned the TDS. It is estimated that utilization of the TDS in the warning decision process could result in $\sim 45$ previously unwarned tornadoes being warned annually, and in $\sim 65$ tornado warnings being issued with a less negative lead time. Examples are shown that demonstrate potential operational usefulness of the TDS.
\end{abstract}

\section{Introduction}

Tornadic debris signatures (TDSs) are well-known features that may be present when a strong wind field lofts debris to the altitude of the radar beam (e.g., Ryzhkov et al. 2005; Schultz et al. 2012a; Bodine et al. 2013; Saari et al. 2014; Van Den Broeke and Jauernic 2014; Van Den Broeke 2015). They are most repeatably characterized by a radial velocity couplet, and by enhanced reflectivity factor at horizontal polarization $\left(\mathrm{Z}_{\mathrm{HH}}\right)$ collocated with depressed co-polar cross-correlation coefficient $\left(\rho_{\mathrm{hv}}\right)$. They may exhibit decreased differential reflectivity $\left(Z_{D R}\right)$ if liquid drops are not present to raise the sample volume's $Z_{D R}$. They are rarely helpful for issuing tornado warnings with positive lead time, because the TDS typically appears several min after tornadogenesis, though light debris may be lofted before the reported tornadogenesis time (e.g., Saari et al. 2014). Considerations for operational use of the TDS are presented by Schultz et al. (2012a, b), and some factors affecting operational use of this signature are discussed by Van Den Broeke (2015).

A lack of spotter reports has been cited as a large contributor to missed tornado warnings (Quoetone et al. 2009); the TDS provides strong evidence that a tornado is occurring or has occurred, which may be difficult to know in certain warning environments (e.g., storms at night or tornadoes embedded in heavy rain). Because many people need additional confirmatory information prior to acting on a tornado warning (e.g., Mileti and Sorenson 1990; Chaney and Weaver 2008; Sherman-Morris 2010; Jauernic and Van Den Broeke 2016), noting a confirmed tornado in warning text may encourage appropriate safety actions.

The tornado warning process and attendant challenges are reviewed by Brotzge and Donner (2013a). A tornado warning is ideally issued prior to tornadogenesis, though in practice many factors may reduce lead time or result in negative lead time or a missed warning. In a large sample of events, it was found that tornado warnings are issued on average 13 min prior to tornadogenesis, though the public would 
like a longer average lead time (Hoekstra et al. 2011). Warnings were issued with negative or zero lead time in $\sim 10 \%$ of events in another study, with negative lead time most common for the first tornado of the day and on days with few tornadoes (Brotzge and Erickson 2009). Storm mode has also been shown to influence tornado warning lead time. Supercell storms are associated with longer lead times, especially if the environment is perceived to be tornado-favorable or the storm's mesocyclone is especially strong (Brotzge et al. 2013b). About 25.5\% of all tornado events during 2003 and 2004 were unwarned (Brotzge et al. 2013b); tornadoes were most likely to be unwarned for the same reasons resulting in negative lead time. Additionally, unwarned tornadoes often occurred at climatologically unfavorable times or were weak (Brotzge and Erickson 2010). The scenarios in which the TDS may provide the greatest benefit to warning operations are the same scenarios in which tornadoes are less likely to be warned or more likely to be associated with negative lead times.

Given the potential value of the TDS in the tornado warning process, it is important to understand how the TDS influences tornado warnings via nowcaster interpretation. This is anticipated to vary through time as nowcasters become better trained and more comfortable with using polarimetric radar variables as they issue warnings. In this study, a baseline is established by examining how the TDS influenced tornado warnings issued during the period of February 2012-May 2013, near the beginning of the polarimetric deployment on the Weather Surveillance Radar-1988 Doppler (WSR-88D) network. Results of this analysis may be compared to those for future time periods, once nowcasters are better trained and more familiar with the use of the TDS. The following specific questions are addressed in this paper:

1) How do temporal offsets between TDSs and reported tornado life cycles vary as a function of estimated tornado intensity, distance to the nearest radar, and land cover?

2) For tornadoes that produced a TDS in the analysis period, what is a typical timeline of TDS occurrence and tornado warning issuance? What factors influence this timeline? How often did tornado warning texts mention radar and observational information for this subset of tornadoes?

3) How are maximum radial velocity difference and spectrum width related to tornadoes and TDSs in this sample, and which signature(s) can best increase nowcaster confidence that a tornado is ongoing?

4) How might utilization of the TDS decrease the number of missed events and reduce negative lead time?

Answering these questions allows the development of some initial suggestions describing how the TDS may be used in the tornado warning process.

\section{Data and methods}

Case selection is described by Van Den Broeke and Jauernic (2014). In summary, all reported tornadoes were identified from 0000 UTC on 1 January 2012 to 0400 UTC on 1 June 2013. This resulted in a database of 1284 tornado events, for which archived WSR88D data were obtained from the National Centers for Environmental Information (NCEI). Events were removed if the data were not polarimetric, if a storm was not present at the expected location, if the tornadoassociated vortex was ill-defined, if volume scans were missing during the tornado time, or if radar data temporal resolution was insufficient to assess the tornado. Poor data quality was also a reason to remove an event - for example, biased $Z_{\mathrm{DR}}$ due to differential attenuation down radial of a hail core or biased $\rho_{\mathrm{hv}}$ due to non-uniform beamfilling at more distant ranges could result in a case being removed from the analysis. A total of 744 tornado events were retained. These were individually analyzed to see if a TDS was present, which was the case for 119 $(16.0 \%)$ of the events. Herein, these 119 TDS events are analyzed alongside the associated tornado warnings. For a detailed discussion of the criteria used to identify a TDS and associated land cover classification, see Van Den Broeke and Jauernic (2014). The same radar data were used to obtain time series of two additional variables that may have value in the tornado warning process:

1) Maximum radial velocity difference $\left(\Delta \mathrm{V}_{\mathrm{r}-\max }\right)$ across the tornado-associated vortex at base scan. This variable was calculated by taking the maximum velocity difference $\left(\Delta \mathrm{V}_{\mathrm{r}}\right)$ in a $2-\mathrm{km}(1.24 \mathrm{mi})$ along-radial by $2^{\circ}$-wide sector containing the tornado. If the tornadoradar distance was $<40 \mathrm{~km}(24.85 \mathrm{mi})$, this was changed to a $2-\mathrm{km}(1.24 \mathrm{mi})$ along-radial by $3^{\circ}$-wide sector to account for the decreasing 
width with decreasing distance of a fixed-angle sector.

2) Maximum spectrum width $\left(\sigma_{v}\right)$ value associated with the base-scan vortex. A value was only recorded if the $\sigma_{\mathrm{v}}$ maximum was well-defined and clearly vortex-associated.

A tornado life cycle was identified for each tornado event, consisting of a genesis time (when the tornado was reported to first occur) and a demise time (when the tornado was reported to dissipate). Reported times of tornado formation and demise may be erroneous, but they were obtained from NCEI's Storm Events Database, representing the most rigorously verified such dataset. Text of tornado warnings and warning updates was obtained for all events from Iowa State University's Iowa Environmental Mesonet (IEM; mesonet.agron.iastate.edu/).

\section{Observations of TDSs relative to tornado life cycles and tornado warnings}

a. Temporal association between tornadoes and accompanying TDSS

The temporal offset between TDS appearance/ disappearance and reported tornado formation/ dissipation was presented by Van Den Broeke and Jauernic (2014); see also their Fig. 3. To summarize, on average across this dataset a TDS appeared $4.4 \mathrm{~min}$ after reported tornadogenesis and dissipated $2.6 \mathrm{~min}$ after reported tornado demise. In $\sim 10 \%$ of events, a TDS-like signature appeared prior to reported tornadogenesis; possible reasons for this are discussed by Saari et al. (2014) and Van Den Broeke (2015). These reasons include possible error in the reported tornadogenesis time, possible light debris lofting in a nontornadic wind field prior to tornadogenesis, possible debris being entrained into the circulation from a prior tornado, and the possible presence of nontornadic vortices. In some cases, a TDS persisted 15-20 min after reported tornado dissipation. Potential factors influencing variability in the timing of TDS appearance relative to tornado formation and dissipation have rarely been reported in the literature. These factors are important to consider, however, if TDSs are to be an important contributor to the tornado warning process.

The time at which a TDS appears relative to the reported tornadogenesis time is primarily a function of the range of the tornado from the radar (Table 1), because beam height increases with range. For TDSs that appeared within $2 \mathrm{~min}$ of tornado formation (including TDSs that appeared prior to tornado formation), the mean tornado-radar distance was approximately $50 \mathrm{~km}(31.07 \mathrm{mi})$. This increased to $\sim 60$ $\mathrm{km}(37.28 \mathrm{mi})$ when a TDS appeared 3-4 min after reported tornado formation, to $\sim 70 \mathrm{~km}(43.50 \mathrm{mi})$ when a TDS appeared 7-10 min after tornado formation, and to nearly $90 \mathrm{~km}(55.92 \mathrm{mi})$ when a TDS appeared $>10$ min after tornado formation (Table 1). Tornado intensity was estimated by EF-scale rating, which represents the maximum damage rating along a tornado's track but which may not be an accurate reflection of the tornado's maximum wind speeds. It less consistently influenced when a TDS appeared. As noted in Van Den Broeke (2015), tornadoes had a higher average EF-scale rating if a TDS appeared prior to reported tornado formation, possibly indicating a stronger antecedent low-level wind field or, in some cases, possibly reflecting debris left over from a prior tornado in a cyclic supercell. Estimated tornado intensity was also generally greater for events in which a TDS appeared well after tornado formation (Table 1). This appeared to be the case because stronger tornadoes tend to last longer, and long-lived tornadoes are capable of producing a TDS many minutes after reported tornadogenesis.

Parent storm mode was biased toward the supercell mode for events in which a TDS appeared well after reported tornado formation, but this appeared to reflect the greater average longevity of mesocycloneassociated tornadoes. Dominant land cover along the tornado track appeared to be a minor contributor to the timing of TDS appearance (Table 1). Grassy land cover was more common for events in which a TDS appears relatively late, possibly reflecting the geographic bias of grassland to areas where supercells and associated longlived tornadoes are most common. Another possible contributor may be lower debris availability from grassy surfaces than from forest. From these results, it appears that tornado-radar distance is sufficient to explain most variability in the time at which a TDS appears relative to reported tornado formation, though the timing of TDS appearance also likely conveys information about tornado intensity.

In contrast, when a TDS disappears relative to reported tornado dissipation is most strongly a function of estimated tornado intensity (Table 2). Intense tornadoes tend to have larger TDSs (Van Den Broeke and Jauernic 2014) and likely loft a larger mean volume of debris, so it is an expected result that a TDS persists longer after a more intense tornado 
Table 1. For several categories representing different appearance times of a TDS after reported tornado formation (min): mean values of tornado EF-scale rating (note that EF-scale rating indicates maximum damage rating achieved along the track), percent of tornadoes produced by supercell storms, mean distance to the nearest WSR-88D, and percentage of tornadoes for which their track was dominated by each of six land cover classifications $(1=$ water; $2=$ urban; $3=$ deciduous forest; $4=$ coniferous forest; $5=$ grass; $6=$ crops). Note that land cover percentages may not add up to $100 \%$ because two co-dominant land cover classifications could be assigned to each tornado event.

\begin{tabular}{|c|c|c|c|c|c|c|c|c|c|}
\hline \multirow{2}{*}{$\begin{array}{r}\text { TDS Appear. after Tornadogenesis } \\
\text { (min) }\end{array}$} & \multirow[t]{2}{*}{$\mathbf{n}$} & \multirow{2}{*}{$\begin{array}{c}\text { Mean EF- } \\
\text { scale Rating }\end{array}$} & \multirow[t]{2}{*}{$\%$ Supercell } & \multirow{2}{*}{$\begin{array}{l}\text { Mean Distance } \\
(\mathbf{k m})\end{array}$} & \multicolumn{5}{|c|}{ Land Cover: \% Each Category } \\
\hline & & & & & 12 & 3 & 4 & 5 & 6 \\
\hline$<0$ (TDS appears before genesis) & 12 & 1.75 & $75 \%$ & 54.8 & $17 \%$ & $25 \%$ & $8 \%$ & $25 \%$ & $42 \%$ \\
\hline 0 (TDS appears at genesis) & 14 & 0.93 & $71 \%$ & 47.7 & $7 \% \quad 21 \%$ & $43 \%$ & $7 \%$ & $29 \%$ & $14 \%$ \\
\hline $1-2$ & 17 & 0.88 & $76 \%$ & 49.6 & $0 \% \quad 24 \%$ & $41 \%$ & $6 \%$ & $29 \%$ & $24 \%$ \\
\hline $3-4$ & 25 & 1.08 & $72 \%$ & 60.5 & $4 \% \quad 12 \%$ & $36 \%$ & $4 \%$ & $28 \%$ & $28 \%$ \\
\hline $5-6$ & 17 & 1.53 & $76 \%$ & 64.2 & $0 \% \quad 18 \%$ & $29 \%$ & $0 \%$ & $47 \%$ & $35 \%$ \\
\hline $7-10$ & 18 & 2.11 & $100 \%$ & 72.0 & $0 \% \quad 6 \%$ & $33 \%$ & $6 \%$ & $44 \%$ & $33 \%$ \\
\hline $11-30$ & 13 & 1.69 & $92 \%$ & 88.6 & $17 \% \quad 8 \%$ & $42 \%$ & $17 \%$ & $42 \%$ & $25 \%$ \\
\hline
\end{tabular}

(Table 2). Tornadoes with which a TDS persisted well after tornado dissipation were more likely to have grassy land cover (Table 2), possibly reflecting the geographic bias of strong tornadoes toward the Great Plains. A higher mean EF-scale rating was also associated with tornadoes in which a TDS disappeared before or at the time of reported tornado dissipation. These events were associated with a larger mean radar-tornado distance (Table 2), so it is speculated that altitude of lofted debris may be an important factor. Land cover did not appear to strongly contribute.

\section{b. Associations between tornado warnings and TDSs}

Among tornadoes with debris signatures, characteristics were examined that might influence the proportion of events being warned, in order to identify particular situations when the TDS might be most useful. These characteristics include storm mode, time of year, and geographic region. An expectation bias may exist under certain conditions, leading to more negative lead time than in other circumstances or missed events altogether. This is common, for instance, with the first tornado of the day (e.g., Brotzge and Erickson 2009), and when tornadoes are produced by non-supercell storms (e.g., Brotzge et al. 2013b).

In this sample of tornadoes with debris signatures, storm mode strongly influenced tornado warning miss rate and when tornado warnings were issued relative to reported tornadogenesis (Table 3). The results presented here for storm mode should be considered preliminary given the small sample sizes of many categories. Tornadoes produced by supercell storms, comprising the majority of those in this dataset, were warned $93.5 \%$ of the time and with an average lead time of $13.7 \mathrm{~min}$. Supercell storms were defined here as organized cells with midlevel (2-6 km [1.24-3.73 mi] above radar level) rotation evident in the $\mathrm{V}_{\mathrm{r}}$ field and coincident with the convective updraft diagnosed using the $Z_{\mathrm{DR}}$ column. TDSs were not produced by other storm modes as frequently (Table 3 ). Those produced by embedded supercells and multicell storms, though not well represented in this dataset, were associated with negative mean lead time. Only one of the five tornadoes from a multicell storm that produced a TDS was warned. Tornadoes produced by linear storm modes were also warned relatively infrequently $(60 \%$ of the time; Table 3). Time of year was an important factor-although mean lead time was high and most tornadoes were warned in the spring, mean lead time was relatively poor in the summer and fall, and many fall tornadoes with TDSs were not warned (Table 3). This is likely because multi-tornado outbreaks in favorable environments and supercell storms occur most often in the spring (e.g., Smith et al. 2012; Fuhrmann et al. 2014).

Geographic region had some influence on tornado warning lead time and percentage of tornadoes warned. Using the regional divisions of Brotzge et al. (2011), there were not enough TDSs in the West region for robust statistical comparison. Elsewhere, however, tornadoes were typically warned in the Great Plains region, with large lead time (Table 3). This may result partially from the large percentage of TDSs associated with supercell tornadoes in this region. Although lead time was comparable in the Southeast, a smaller percentage of TDS-producing tornadoes was warned, possibly because linear storm modes were more common. In the Midwest/East region, lead time was relatively small and only $73.3 \%$ of tornadoes with TDSs were warned 
Table 2. As for Table 1, except for several categories representing different disappearance times of a TDS relative to reported tornado dissipation.

\begin{tabular}{|c|c|c|c|c|c|}
\hline TDS Disapp. after Tornado Demise & $\mathbf{n}$ & Mean EF- & $\%$ Supercell & Mean Distance & Land Cover: \% Each Category \\
\hline (min) & & scale Rating & & $(\mathrm{km})$ & $\begin{array}{llllll}1 & 2 & 3 & 4 & 5 & 6\end{array}$ \\
\hline$<0$ (TDS gone before dissipation) & 24 & 1.63 & $83 \%$ & 74.2 & $0 \% \quad 33 \% \quad 25 \%$ \\
\hline 0 (TDS gone at dissipation) & 10 & 1.50 & $70 \%$ & 39.2 & $\begin{array}{lll}0 \% & 11 \% & 33 \% \\
\end{array}$ \\
\hline $1-3$ & 18 & 1.22 & $94 \%$ & 66.7 & $\begin{array}{llll}0 \% & 33 \% & 39 \% \\
\end{array}$ \\
\hline $4-5$ & 20 & 1.15 & $65 \%$ & 50.2 & $\begin{array}{llllll}0 \% & 35 \% & 30 \% & 15 \% & 25 \% & 25 \% \\
\end{array}$ \\
\hline $6-10$ & 14 & 1.57 & $79 \%$ & 62.1 & $\begin{array}{llllll}7 \% & 7 \% & 43 \% & 14 \% & 50 \% & 21 \% \\
\end{array}$ \\
\hline $11-21$ & 11 & 1.64 & $82 \%$ & 61.8 & $\begin{array}{cccccc}9 \% & 27 \% & 27 \% & 0 \% & 64 \% & 9 \%\end{array}$ \\
\hline
\end{tabular}

Table 3. Tornado warning lead time for the sample of TDS-producing tornadoes by storm mode, season, and geographic region. The column '\% Warned' denotes the percentage of tornado events in each category that were associated with a tornado warning. ' $\%$ Supercell' denotes the percentage of tornadoes in each category that were associated with the supercell storm mode.

\begin{tabular}{|c|c|c|c|c|}
\hline \multicolumn{5}{|l|}{ Lead Time by Storm Mode } \\
\hline Mode & $\mathbf{n}$ & Mean Lead Time (min) & $\%$ Warned & \\
\hline Supercell & 92 & 13.71 & $93.5 \%$ & \\
\hline Linear & 15 & 12.90 & $60.0 \%$ & \\
\hline Multicell & 5 & -5.00 & $20.0 \%$ & \\
\hline Embedded Supercell & 4 & -1.33 & $75.0 \%$ & \\
\hline Landspout & 3 & -3.67 & $100.0 \%$ & \\
\hline \multicolumn{5}{|l|}{ Lead Time by Season } \\
\hline Season & $\mathbf{n}$ & Mean Lead Time (min) & \% Warned & $\%$ Supercell \\
\hline SPR: Mar-May & 76 & 15.79 & $92.1 \%$ & $82.9 \%$ \\
\hline SUM: Jun-Aug & 17 & 2.67 & $88.2 \%$ & $82.4 \%$ \\
\hline FALL: Sept-Nov & 11 & 2.80 & $45.5 \%$ & $72.7 \%$ \\
\hline WIN: Dec-Feb & 15 & 9.25 & $80.0 \%$ & $73.3 \%$ \\
\hline \multicolumn{5}{|c|}{ Lead Time by Geographic Region } \\
\hline Region & $\mathbf{n}$ & Mean Lead Time (min) & \% Warned & $\%$ Supercell \\
\hline Plains & 58 & 14.71 & $94.8 \%$ & $87.9 \%$ \\
\hline Southeast & 44 & 12.23 & $79.6 \%$ & $77.3 \%$ \\
\hline Midwest/East & 15 & 3.55 & $73.3 \%$ & $60.0 \%$ \\
\hline West & 2 & -6.00 & $50.0 \%$ & $100.0 \%$ \\
\hline
\end{tabular}

(Table 3). These results suggest that the use of the TDS is most likely to improve tornado warnings in the Midwest/East region, during the fall season, and for tornadoes produced by multicell and linear convective modes.

The utilization of the TDS in tornado warning and warning update text was also investigated in comparison with several other factors that are sometimes mentioned. These factors include other radar metrics such as rotation, visual confirmation of a tornado, mention of a storm's tornadic history, and damage/injuries. Statistics presented here are valid for the 102 tornado events for which a TDS was observed and a tornado warning was issued. The remaining 17 tornado events included in this study that produced a TDS were unwarned.

The initial tornado warning text, defined as the first warning for a particular tornado, rarely included mention of a TDS ( $n=2 ; 2.0 \%$; Table 4). Likewise, damage/injuries and a storm's tornadic history were rarely mentioned in the initial warning (Table 4). Visual confirmation was noted more often $(\mathrm{n}=23 ; 22.5 \%)$, although radar metrics such as rotation were noted most commonly $(\mathrm{n}=85 ; 83.3 \%)$. When the text of warning updates is included in a similar analysis, the TDS was mentioned for $22.5 \%$ of events $(n=23$; Table 4$)$. Strong increases in the frequency of mention are also noted (Table 4) for visual confirmation (noted for nearly $61 \%$ of events), for damage or injuries (noted for nearly $20 \%$ of events), and for the tornadic history of the storm (noted for $22.5 \%$ of events). This is because visual confirmation of a tornado and resulting damage and/ or injuries are most likely after the tornado has been present for some time.

Tornadoes for which the initial warning included mention of a TDS are associated with shorter mean lead times than other events (Table 4). The mean lead 
Table 4. Percentage of TDS-producing tornado events for which the initial warning contained mention of a given factor ('Initial Warning'), for which the initial warning or any subsequent warning update contained mention of a given factor ('Any Warning'), and mean lead time if the initial tornado warning contained mention of a given factor ('Mean Lead').

\begin{tabular}{|r|c|c|c|}
\hline Factor Mentioned in Warning Text & Initial Warning (\%) & Any Warning (\%) & Mean Lead (min) \\
\hline TDS & $2.0 \%$ & $22.5 \%$ & -2.50 \\
\hline Other Radar Metric(s) & $83.3 \%$ & $93.1 \%$ & 12.12 \\
\hline Visual Confirmation & $22.5 \%$ & $60.8 \%$ & 17.04 \\
\hline Damage/Injuries & $5.9 \%$ & $19.6 \%$ & 13.50 \\
\hline Tornadic Storm History & $9.8 \%$ & $22.5 \%$ & 18.50 \\
\hline
\end{tabular}

time for all events included in this study was $12.5 \mathrm{~min}$, comparable to the national average value of $13 \mathrm{~min}$ (Hoekstra et al. 2011). Mean lead time if the initial warning contained mention of other radar metrics such as rotation was comparable to the national average, at $12.1 \mathrm{~min}$. Mean lead time was higher than average if visual confirmation was noted (17.0 min, representing observations of a prior tornado from the same storm), and if a storm's tornadic history was noted (18.5 min). In contrast, lead times for the 2 events in which the TDS was initially mentioned were $3 \mathrm{~min}$ and -8 min (mean lead time $-2.5 \mathrm{~min}$ ). In the event with a 3 -min lead time, a prior tornado from the same storm produced the TDS. In the event with the 8-min negative lead time, no other factors were mentioned in the text of the initial tornado warning, making it possible that a tornado warning would not have been issued as soon had the TDS not appeared. A TDS was first mentioned, on average, $31.1 \mathrm{~min}$ after the initial tornado warning was issued, and first mentioned $11.3 \mathrm{~min}$ after reported tornadogenesis. This was longer than the average of 4.4 min between reported tornadogenesis and appearance of the TDS noted by Van Den Broeke (2015) for a highly overlapping sample of TDS-producing tornadoes.

The TDS was mentioned in several ways among this sample of tornado warnings and warning updates. Most commonly, the warning mentioned radar confirmation of a tornado (14 occurrences; Table 5). A debris signature was specified in five events, and the possibility of debris was mentioned in four additional events without mention of a 'debris signature' (Table 5). One especially clear inclusion of the TDS in a warning text stated that "Doppler radar has a tornado debris signature on this storm...confidence in a tornado is very high at this time." Especially early in the utilization of the TDS when much of the public may not have understood the term 'tornado debris signature,' this statement clearly indicates the implication of the debris signature. Further work could assess how the public would like the TDS to be used in future tornado warning text.

\section{c. Other tornado indicators}

A TDS is associated with rotation evident in the radial velocity $\left(\mathrm{V}_{\mathrm{r}}\right.$ ) field (e.g., Ryzhkov et al. 2005; Schultz et al. 2012a; Van Den Broeke and Jauernic 2014; Smith et al. 2015). A tornadic circulation is also frequently associated with a local maximum in the $\sigma_{v}$ field (e.g., Yu et al. 2007; Spoden et al. 2012). Thus, $\Delta \mathrm{V}_{\mathrm{r}-\max }$ and $\sigma_{\mathrm{v}}$ are next investigated as variables that potentially add confidence to the presence or absence of a tornado among events in this dataset. Also, characteristics of $\Delta \mathrm{V}_{\mathrm{r}-\max }$ and $\sigma_{\mathrm{v}}$ are related to times when the TDS is present and absent.

The $\Delta \mathrm{V}_{\mathrm{r}-\max }$ across a vortex is used operationally to assess vortex strength. In a tornado detection algorithm (TDA) developed by the National Severe Storms Laboratory (NSSL), a minimum $\Delta \mathrm{V}_{\mathrm{r}}$ of $11 \mathrm{~m} \mathrm{~s}^{-1}$ between an azimuthally adjacent velocity gate pair is required to be considered of potential interest (Mitchell et al. 1998). Additionally, $\sim 3 \%$ of mesocyclones detected by NSSL's mesocyclone detection algorithm (MDA) are associated with tornadoes, and most missed tornadoes are EF-0 or occasionally EF-1 (Jones et al. 2004). This indicates that strong tornadoes are likely to be associated with a relatively strong $\Delta \mathrm{V}_{\mathrm{r}}$ values, presumably making them easier to detect using traditional radar methods. An adjacent gate pair is not required to indicate a vortex for a TDS to be possible, but a more broad circulation may be TDS-associated. Generally, the required strength of this circulation has not been defined in the literature (e.g., Van Den Broeke and Jauernic 2014). Here, relationships of $\Delta \mathrm{V}_{\mathrm{r}}$ values to the TDS life cycle are presented.

Tornado events were sorted into three groups of roughly equal size by reported longevity. The top third consisted of tornadoes reported to occur for $>15 \mathrm{~min}$, and the bottom third for tornadoes reported to occur for $<7 \mathrm{~min}$. For events in the top third of longevity (most of which 
Table 5. Mention of the TDS in tornado warning and warning update text.

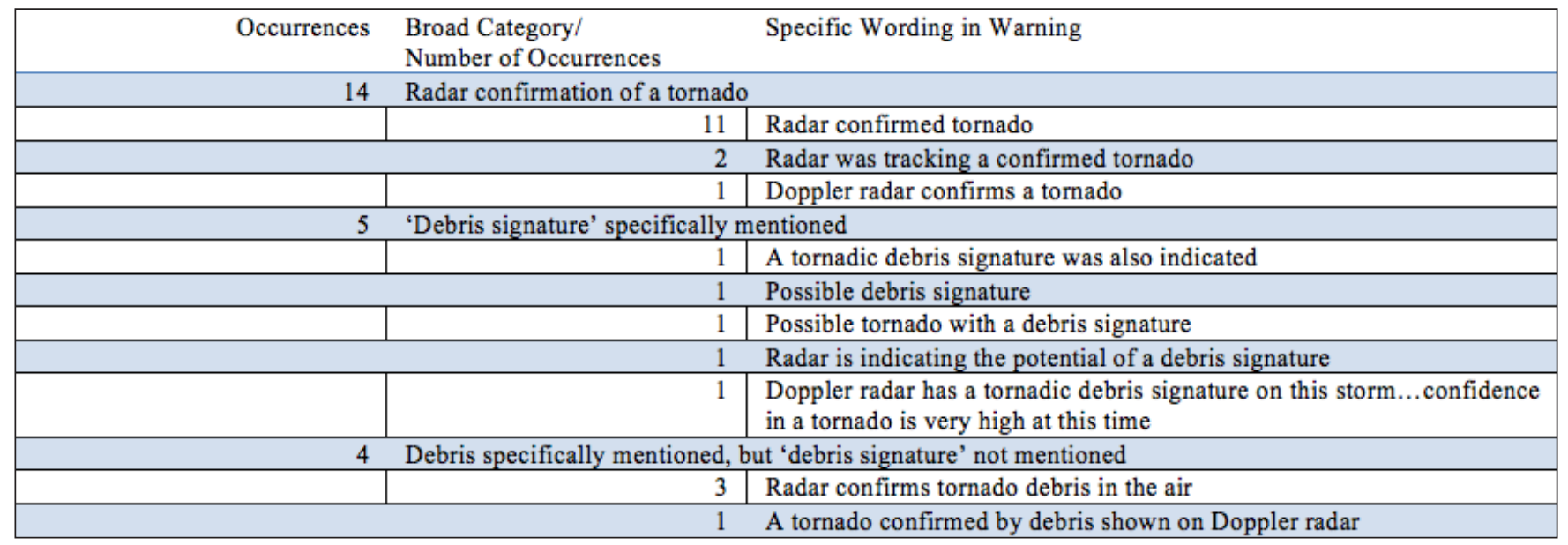

were strong tornadoes), mean $\Delta \mathrm{V}_{\mathrm{r}-\max }$ was $100.5 \mathrm{~m} \mathrm{~s}^{-1}$ at times when a TDS was present and $80.2 \mathrm{~m} \mathrm{~s}^{-1}$ when a TDS was not present. Using a Wilcoxon-Mann-Whitney test (e.g., Corder and Foreman 2014), this difference was significant $(p=0.006$; Table 6$)$. For tornadoes in the middle and lower third of longevity, the difference between TDS and non-TDS times was not statistically significant (Table 6). This result indicates that $\Delta \mathrm{V}_{\mathrm{r} \text {-max }}$ and the TDS are likely to convey similar information for stronger, long-lived tornadoes, but this may not be the case for shorter-lived tornadoes. A TDS-like signature combined with a large $\Delta \mathrm{V}_{\mathrm{r}}$ value is strong evidence for an ongoing tornado.

Among the 68 events for which a radar sample volume representative of both tornado genesis and demise times was available, $\Delta \mathrm{V}_{\mathrm{r}}$ at the time of genesis exceeded that at the time of demise in $80.9 \%$ of events, and the genesis $\Delta \mathrm{V}_{\mathrm{r}}$ exceeded the demise value by $>5.14 \mathrm{~m} \mathrm{~s}^{-1}$ (10 kt.) in $67.6 \%$ of events. Times representative of genesis and demise were required to be within $2 \mathrm{~min}$ of the reported genesis/demise time. It is here hypothesized that time change of $\Delta \mathrm{V}_{\mathrm{r}}$ should be positive at the genesis time and negative at the demise time, assuming a broad-scale circulation associated with a tornado that is intensifying at tornadogenesis and weakening at demise. This was the case for $64.1 \%$ of genesis times and $79.7 \%$ of demise times. The temporal trend of $\Delta \mathrm{V}_{\mathrm{r}}$ was strongest and most repeatable across demise times because $\Delta \mathrm{V}_{\mathrm{r}}$ was sometimes small around the time of tornadogenesis, especially for weak tornadoes. Mean $\Delta \mathrm{V}_{\mathrm{r}}$ was $44.2 \mathrm{~m} \mathrm{~s}^{-1}$ for all genesis times and $32.2 \mathrm{~m} \mathrm{~s}^{-1}$ for all demise times.

Time change in $\Delta \mathrm{V}_{\mathrm{r}}$, defined as the change from the prior radar sample volume to the sample at genesis or demise, averaged $+7.4 \mathrm{~m} \mathrm{~s}^{-1}$ for genesis times and $-12.0 \mathrm{~m} \mathrm{~s}^{-1}$ for demise times. Thus, temporal trend of $\Delta \mathrm{V}_{\mathrm{r}}$ may have some value in diagnosing tornado genesis and demise times. Though the magnitude of these values is not large relative to the sample of all $\Delta \mathrm{V}_{\mathrm{r}}$ values, a change in the sign of the time rate of change of $\Delta \mathrm{V}_{\mathrm{r}}$ (i.e., $\Delta \mathrm{V}_{\mathrm{r}} / \Delta \mathrm{t}$ ) can be an indicator of increasing or decreasing tornado potential when combined with other signatures (e.g., a TDS, the $\mathrm{V}_{\mathrm{r}}$ value, the $\sigma_{\mathrm{v}}$ value). Particularly, when $\Delta \mathrm{V}_{\mathrm{r}} / \Delta \mathrm{t}$ is increasing, the appearance of an associated TDS-like signature should be taken as in indication that a tornado may be in progress. Temporal trend of $\Delta \mathrm{V}_{\mathrm{r}}$ was significantly different between TDS and non-TDS times for all tornadoes (Table 6). During TDS times, mean $\Delta \mathrm{V} / \Delta \mathrm{t}$ was typically negative, although it was typically less negative or positive during non-TDS times. This finding reflects the bias of the TDS to be present during and after the time of tornado demise when the associated circulation is weakening, rather than during the time of tornadogenesis when the associated circulation is strengthening.

Spectrum width has also been associated with tornadic circulations. In combination with other variables, $\sigma_{v}$ contributes valuable information to the tornado warning process (e.g., Yu et al. 2007; Spoden et al. 2012). Operationally, a $\sigma_{v}$ threshold of $10.3 \mathrm{~m} \mathrm{~s}^{-1}$ has been suggested as a typical minimum for a tornadic circulation, and $\sigma_{v}$ maxima have been observed to sometimes precede tornadogenesis and thus increase confidence for issuing a tornado warning (Spoden et al. 2012). One key limitation of $\sigma_{v}$ seen in this study was that a well-defined maximum was not associated with the tornadic circulation in a large number of events. Another important limitation of $\sigma_{v}$ was the discrete nature of 
Table 6. Other tornado indicators $\left(\Delta \mathrm{V}_{\mathrm{r}-\max }, \Delta \mathrm{V}_{\mathrm{r}-\mathrm{max}} / \Delta \mathrm{t}\right.$, and $\left.\sigma_{\mathrm{v}}\right)$ related to the TDS for the approximate top, middle, and lower thirds of reported tornado longevity ( $\mathrm{min})$. 'Top third' were tornadoes reported for $>15$ min, 'middle third' were tornadoes reported for 7-15 min, and 'lower third' were tornadoes reported for $<7 \mathrm{~min}$. Top three rows indicate the percentage of events for which the mean value of an indicator was greater at times with a TDS than for times without a TDS. Wilcoxon-Mann-Whitney p-values $<0.05$ (indicated by bold/italic font) indicate that the population of a given indicator was statistically different between times with and without a TDS.

\begin{tabular}{|c|c|c|c|c|}
\hline & & Top Third & Middle Third & Lower Third \\
\hline \multirow[t]{2}{*}{ TDS $>$ Non-TDS } & $\Delta \mathrm{V}_{\mathrm{r} \cdot \max }$ & $70.0 \%$ & $54.8 \%$ & $34.3 \%$ \\
\hline & $\Delta \mathrm{V}_{\mathrm{r} \cdot \max } / \Delta \mathrm{t}$ & $10.0 \%$ & $19.0 \%$ & $8.6 \%$ \\
\hline \multicolumn{2}{|r|}{$\sigma_{\mathrm{v} \cdot \max }$} & $55.0 \%$ & $57.1 \%$ & $17.1 \%$ \\
\hline \multirow[t]{3}{*}{$\Delta \mathrm{V}_{\mathrm{r} \cdot \max }\left(\mathrm{m} \mathrm{s}^{-1}\right)$} & Mean value, TDS & 100.54 & 78.10 & 54.96 \\
\hline & Mean value, non-TDS & 80.20 & 72.67 & 61.17 \\
\hline & MWM $p$-value & 0.006 & 0.362 & 0.082 \\
\hline \multirow{3}{*}{$\Delta \mathrm{V}_{\mathrm{r} \cdot \max } / \Delta \mathrm{t}\left(\mathrm{m} \mathrm{s}^{-2}\right)$} & Mean value, TDS & -4.13 & -8.88 & -10.04 \\
\hline & Mean value, non-TDS & 9.53 & 1.84 & 4.40 \\
\hline & MWM $p$-value & 0.000 & 0.005 & 0.002 \\
\hline \multirow[t]{3}{*}{$\sigma_{\mathrm{v} \cdot \max }\left(\mathrm{m} \mathrm{s}^{-1}\right)$} & Mean value, TDS & 29.22 & 27.43 & 22.28 \\
\hline & Mean value, non-TDS & 28.13 & 25.97 & 24.87 \\
\hline & MWM $_{p \text {-value }}$ & 0.025 & 0.284 & 0.035 \\
\hline
\end{tabular}

pixel values $-\sigma_{\mathrm{v}}$ values in a large range are reported as the same value, and for a given radar scanning strategy, the $\sigma_{v}$ value is "maxed out" at a particular value that is often exceeded in strong vortices. Thus, $\sigma_{v}$ variations across the tornado life cycle were not shown as well as what would be ideal for a nowcaster.

The vortex-associated $\sigma_{\mathrm{y}}$ value was slightly higher when a TDS was ongoing for tornadoes in the top two-thirds of longevity, though the opposite was true for shorter-lived tornadoes (Table 6). Though the differences were significant for relatively short- and long-lived tornadoes (Table 6), the small magnitude of mean difference indicates that this result may be difficult to implement operationally. In those events for which a $\sigma_{\mathrm{v}}$ maximum was well-defined $(\mathrm{n}=54), \sigma_{\mathrm{v}}$ was larger on average at the genesis time than at the demise time in $48.1 \%$ of events. An additional $29.6 \%$ of events had the same $\sigma_{\mathrm{v}}$ value at the genesis and demise times, and $22.2 \%$ of events had larger $\sigma_{v}$ at demise than at genesis. Notably, genesis $\sigma_{v}$ exceeded the mean $\sigma_{v}$ value across the entire tornado life cycle for $56.7 \%$ of events, and demise $\sigma_{\mathrm{v}}$ was lower than the mean value for $50.8 \%$ of events. These results indicate that $\sigma_{\mathrm{v}}$ temporal fluctuations have value in diagnosing the tornado life cycle for many events.

\section{d. Potential for improved tornado warnings}

The TDS typically first appears several min after tornadogenesis (e.g., Van Den Broeke 2015), limiting its usefulness in the warning process. Because many TDSs appear within 5 min of tornadogenesis, however, they may be a valuable source of information during events that would otherwise go undetected. Improvement to tornado warnings should be greatest for events that are currently missed and for which a warning is issued with negative lead time. Here, these categories of events are examined to assess how much improvement might be realized by using the TDS in the warning process. Among this sample of tornadoes with a TDS, 17 were unwarned and 25 had negative lead time, in total representing $35.3 \%$ of the sample. Figure 1 illustrates the EF-scale ratings of events in each category. Note that many tornadoes in this sample for which warnings were missed or had negative lead time were EF-1 and EF-2 (Fig. 1), which is thought to be the case because TDSs are relatively uncommon for EF-0 events (e.g., Van Den Broeke and Jauernic 2014). Thus, the TDS may be most likely to improve warnings for missed EF$1+$ events, which are especially important to warn.

In the subset of events with no tornado warning $(n=17)$, the nature of events clearly contributed. Only four of these events had a well-defined $\Delta \mathrm{V}_{\mathrm{r}}$ and $\sigma_{\mathrm{v}}$ maximum at the time of tornadogenesis. Nevertheless, the polarimetric variables were of sufficient quality to identify a TDS. Among the sample of unwarned tornadoes, a TDS appeared on average only $2.59 \mathrm{~min}$ post-tornadogenesis and persisted for an average of 7.53 min (approximately three 'base scans' when SAILS or MESO-SAILS is used to provide intra-volume base scans). In seven of the events, the TDS persisted for at least $9 \mathrm{~min}$. In only two of the events did a TDS appear $>5$ min after tornadogenesis. Thus, in 15 of 17 of these events ( $88 \%)$, a tornado warning could have been issued 


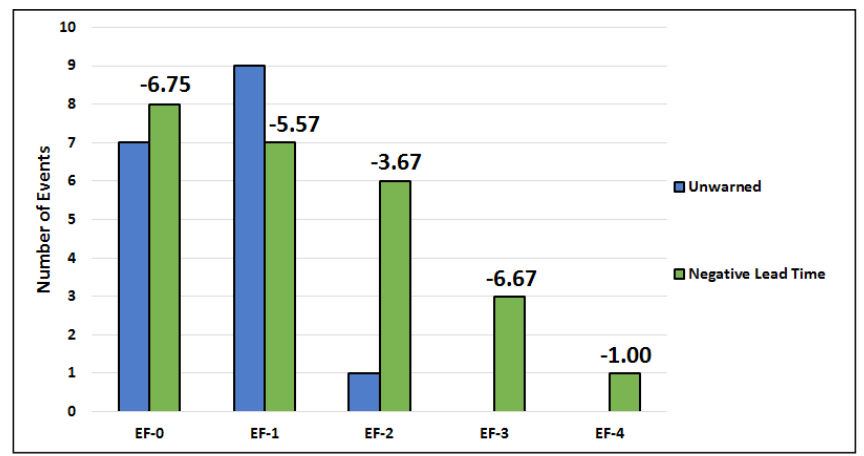

Figure 1. Intensity categories of the tornado events in this dataset that were unwarned (blue bars) or had negative lead time (green bars). Numbers above the green bars are mean lead time ( $\mathrm{min}$ ) for each intensity category. Click image for an external version; this applies to all figures hereafter.

shortly after tornadogenesis.

Two challenging warning scenarios have been selected to illustrate the use of the TDS to raise confidence in the need to issue a tornado warning. The first case occurred in October 2012 in California, outside the period of climatologically high California tornado occurrence. This tornado was rated EF-1 and persisted for $2 \mathrm{~min}$. A TDS-like signature appeared 3 min prior to reported tornadogenesis, possibly due to error in the reported genesis time. At the time of reported tornadogenesis, a thunderstorm with an echo appendage was located $\sim 62 \mathrm{~km}(38.53 \mathrm{mi})$ from the radar location (Fig. 2a). The storm was weakly supercellular, evidenced by weak rotation (shown here at base scan; Fig. 2b). A $\sigma_{v}$ maximum was collocated with the vortex, but the magnitude of this maximum was below the threshold identified by Spoden et al. (2012) as typically occurring in tornadic circulations (Fig. 2c). A TDS was visible as a region of near-zero $Z_{\mathrm{DR}}$ (Fig. $2 \mathrm{~d}$ ) and $\rho_{\mathrm{hv}}$ suppressed as low as 0.59 (mean value 0.788 ; Fig. 2e) collocated with a mostly isolated area of $\mathrm{Z}_{\mathrm{HH}}>25 \mathrm{dBZ}$ on the storm's southwestern side (TDS inside the white oval in Fig. 2). In this case, confidence in an out-of-season tornado could be increased by the presence of a TDS, especially given the presence of base-scan rotation, slightly increased $\sigma_{\mathrm{v}}$, and a collocated $\mathrm{Z}_{\mathrm{HH}}$ maximum. This combination of characteristics, including a vortex at a tornado-favorable storm-relative location and polarimetric signatures that are often associated with a TDS, does not appear to be often seen apart from debris lofting. Nevertheless, there is always a potential for false alarms, especially in such events where the TDSlike signature is marginal.
In a second event, that occurred in Pennsylvania during October, a long-duration (25 min) EF-1 tornado occurred at large range from the nearest WSR-88D radar. Debris was apparent in association with the storm's mesocyclone for $44 \mathrm{~min}$ starting $1 \mathrm{~min}$ posttornadogenesis. Near the time of tornadogenesis, the storm was at a range of $\sim 190 \mathrm{~km}(118.06 \mathrm{mi})$ from the observing radar, and in $\mathrm{Z}_{\mathrm{HH}}$ did not appear especially different from nearby storms (Fig. 3a). The storm was weakly supercellular given modest updraft rotation (e.g., Fig. 3b), though no strong rotational signature was apparent through the tornado's life cycle. Spectrum width was slightly elevated in the area of rotation (Fig. $3 c$ ), though this maximum was not focused on a particular location and was typically not of large enough magnitude to meet the threshold of Spoden et al. (2012). Thus, given the base variables, little was present to indicate a tornado, likely due to the large range. Differential reflectivity was generally near $0 \mathrm{~dB}$ over most of the region (Fig. $3 \mathrm{~d}$ ) given the large altitude $(\sim 3.9 \mathrm{~km}[2.42$ mi] altitude at base scan) and dominance of ice-phase scatterers, so it is not particularly instructive. A welldefined minimum in correlation coefficient, however, is collocated with the mesocyclone with values too low to be associated with meteorological scatter (minimum value 0.53 ; areal average value 0.84 ; Fig. $3 \mathrm{e}$ ). These very low correlation coefficient values persist for 44 min and fill the mesocyclone. No other storm in the region looks similar (Fig. 4), which precludes most data quality effects and the possibility that mixed-phase precipitation is the dominant contributor (which is also precluded by the magnitude of minimum $\rho_{\mathrm{hv}}$ values, e.g., WDTD 2016). Presence of the signature solely due to hail is also precluded, because $\rho_{\mathrm{hv}}$ in hail rarely drops below 0.75 over large areas (WDTD 2016) and nearby storms clearly contained hail but did not exhibit a similar signature (Fig. 4). In this case, given the strikingly different appearance of the storm over a long duration and the presence of $\rho_{\mathrm{hv}}$ values $<0.6$ in an area of apparently good data, it was probable that debris was being lofted by the storm, and a tornado warning seems warranted. Though EF-1 tornadoes rarely loft debris visible at this range (e.g., Van Den Broeke and Jauernic 2014), the long duration of the tornado (25 min) and the time of year (October, when large quantities of readilylofted leaves are present) point to an unusual event in which debris was diffused throughout much of the mesocyclone.

Tornadoes for which a warning was issued with negative lead time were also a focus. These 25 events had 


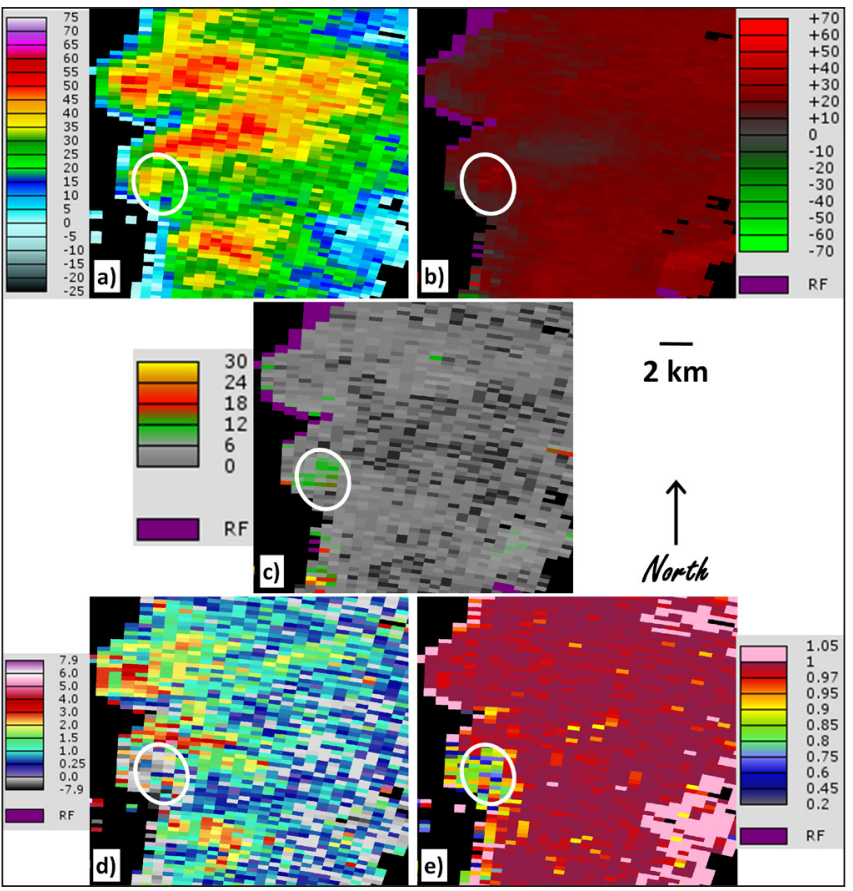

Figure 2. Example of a TDS at the $0.5^{\circ}$ elevation angle from KDAX (Sacramento, California) at 2203 UTC on 22 October 2012. (a) is reflectivity factor (dBZ), (b) is radial velocity (kt), (c) is spectrum width (kt), (d) is differential reflectivity $(\mathrm{dB})$, and (e) is correlation coefficient. The white oval indicates the TDS; beam centerline near the center of the white oval is at an altitude of $\sim 0.79 \mathrm{~km}(0.49 \mathrm{mi}$; range from KDAX $\sim 62$ $\mathrm{km}[38.53 \mathrm{mi}])$.

an average lead time of $-5.44 \mathrm{~min}$. They were typically associated with a larger $\Delta \mathrm{V}_{\mathrm{r}}$ and more pronounced $\sigma_{v}$ maximum than events for which no warning was issued. Of these events, a TDS was observed before the warning in 13 cases $(52.0 \%)$. Average time from TDS appearance to tornado warning issuance was $3.46 \mathrm{~min}$ for these 13 events.

\section{Summary and discussion}

In the first 16 months of widespread polarimetric radar data collection, the TDS was mentioned in tornado warning text in $\sim 22.5 \%$ of TDS events, usually in a warning update. Use of the TDS in tornado warning text most commonly took the form of speaking of a 'radar confirmed tornado.' This wording conveys certainty in the presence of a tornado. Another set of warnings specifically mentioned a 'debris signature' or the presence of debris as indicated by the radar. Nowcasters are encouraged to convey the implication of the TDS

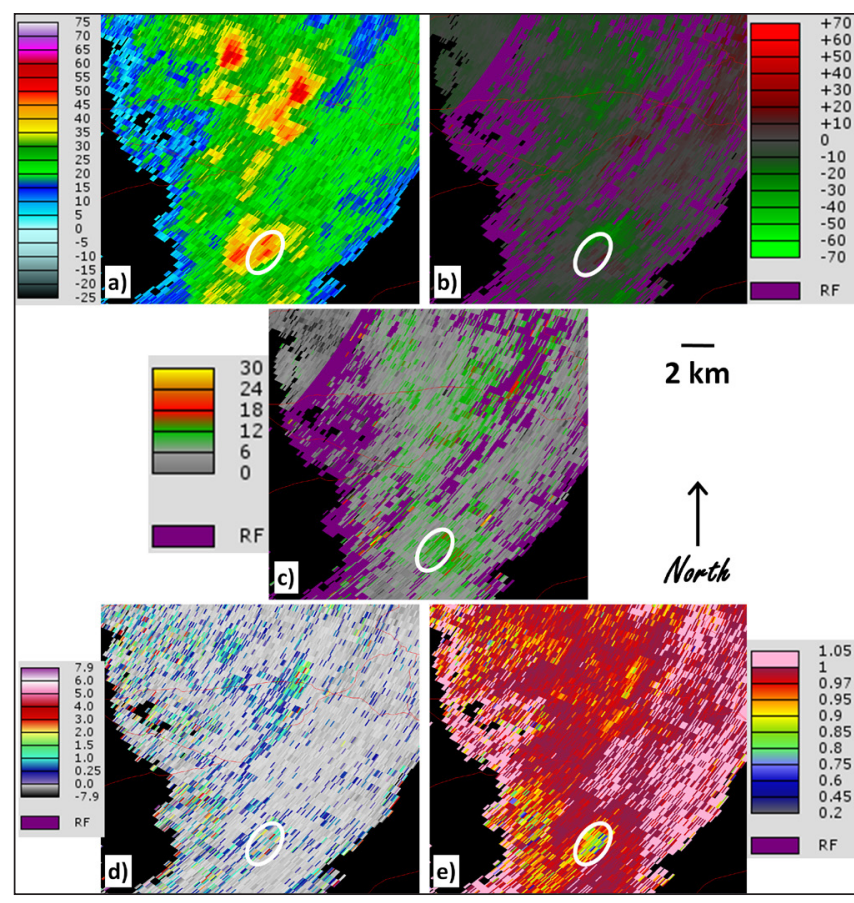

Figure 3. As in Fig. 2, except for data at the $0.5^{\circ}$ elevation angle from KCCX (State College, Pennsylvania) at 0001 UTC on 20 October 2012. The center of the white oval is at an altitude of approximately $3.93 \mathrm{~km}(2.44$ mi; range from KCCX $\sim 190 \mathrm{~km}$ [118.06 mi]).

in warning text (e.g., 'confidence in a tornado is very high').

Tornadoes have a general tendency to be unwarned or to have negative lead time in repeatable circumstances, including the first tornado of the day and in the climatologically unfavorable season. Appearance of a TDS, if used by nowcasters, may result in fewer missed tornado warnings and a reduction in the magnitude of negative lead time for some warnings. This improvement is less likely to be realized for tornadoes far from the observing radar, because it takes longer on average for the TDS to appear in those events. The TDS is most likely to improve tornado warnings for events in the cool season and outside of the geographic region where tornado outbreaks are climatologically common. The TDS may provide special benefit for non-supercell tornadoes, though they do not commonly produce a TDS. Nowcasters should also be aware that a TDS often persists after the time of reported tornado demise (e.g., Van Den Broeke 2015; Houser et al. 2016), so a tornado may no longer be ongoing if a TDS is present. In this case, decreasing $\sigma_{v}$ values associated with the circulation and TDS have some value to indicate tornado weakening or demise. If other signatures occur 


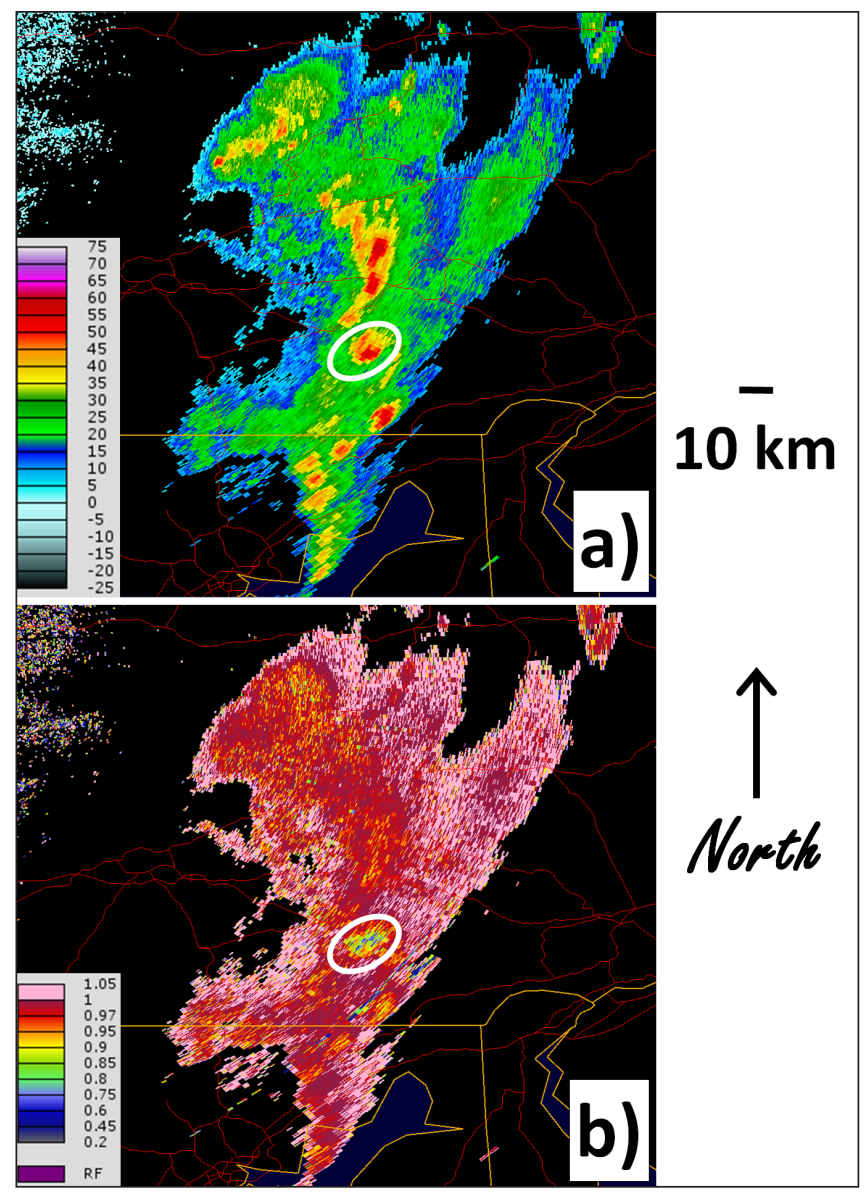

Figure 4. (a) as in Fig. 2a and (b) as in Fig. 2e, except for data at the $0.5^{\circ}$ elevation angle from KCCX (State College, Pennsylvania) at 0016 UTC on 20 October 2012. The center of the white oval is at an altitude of approximately $3.80 \mathrm{~km}(2.36 \mathrm{mi}$; range from KCCX $\sim 186 \mathrm{~km}[115.58 \mathrm{mi}])$.

such as rotation in the $\mathrm{V}_{\mathrm{r}}$ field, and particularly a large nearly-collocated $\Delta \mathrm{V}_{\mathrm{r}}$ value, nowcasters are encouraged to retain a tornado warning as long as a TDS is visible. Decreasing $\Delta \mathrm{V}_{\mathrm{r}}$ values in the presence of a TDS may indicate that tornado demise is imminent or occurring, but this is not universal among cases examined.

$\Delta \mathrm{V}_{\mathrm{r}}$ was more robust than $\sigma_{\mathrm{v}}$ among this sample of tornadoes for diagnosing tornado genesis and demise times. Both the magnitude of $\Delta \mathrm{V}_{\mathrm{r}}$ and the temporal change of $\Delta \mathrm{V}_{\mathrm{r}}$ from one sample volume to the next showed greater ability to infer tornado genesis or demise than the magnitude of $\sigma_{\mathrm{v}}$. $\sigma_{\mathrm{v}}$ was also less likely to be available at a particular analysis time and often did not show a defined maximum at the location of the tornadic circulation, but nevertheless its temporal trends were found to have some potential value. To get the best operational utility out of $\Delta \mathrm{V}_{\mathrm{r}}$, it would be ideal to normalize the $\Delta \mathrm{V}_{\mathrm{r}}$ value by the time between successive sample volumes. This would negate the effect of different sample times associated with different volume coverage patterns. For operational use, one key weakness of $\Delta \mathrm{V}_{\mathrm{r}}$ is that values are often not of large magnitude for short-lived, weak tornadoes. It is also important to note that WSR-88D radars rarely resolve the tornado itself, but rather the larger-scale circulation with which the tornado is associated. In this analysis, there were some cases in which the velocity couplets from two successive tornadoes blended, even if those tornadoes were separated by several minutes. These events were excluded from the analysis described here. In these cases, $\Delta \mathrm{V}_{\mathrm{r}}$ may provide little warning of a new tornado, though this may be unimportant because a tornado warning should generally be continuously in place for a rapidly cycling storm.

When a new component is introduced to the workflow of issuing tornado warnings, there is potential to increase the false alarm rate (FAR). This is particularly true with the TDS, as it is not always clear what to do when a marginal TDS-like signature overlaps only marginal indicators in $\mathrm{V}_{\mathrm{r}}$ and $\sigma_{\mathrm{v}}$. A marginal TDS-like signature may appear near the radar site when clutter and/or bioscatter are present. In this case, a combination of radar parameters and situational knowledge must be used to distinguish a true TDS. Nonuniform beam filling can cause a similar signature, but not usually at the range of most true TDSs. In any case, the nowcaster should incorporate situational awareness and spotter information when possible to reduce the occurrence of false alarms due to marginal TDS-like signatures. Despite the potential for an increased FAR, because $\mathrm{V}_{\mathrm{r}}$ and $\sigma_{\mathrm{v}}$ products often do not indicate a tornadic circulation (particularly in historic cases that were unwarned or had negative lead time), nowcasters are encouraged to utilize the TDS in addition to other factors considered in the warning decision process.

These results indicate that utilizing the TDS in the tornado warning process could result in improved warnings for many of those events in which a tornado is unwarned, and for approximately half of events with negative lead times. For the former, this improvement consists of issuing a warning for some tornadoes that were missed prior-even if the warning is issued with negative lead time, as will be the case most of the time, this is an improvement from issuing no warning. For the latter, improvement consists of reducing the magnitude of negative lead time. Quantitatively, for these negative lead-time events a TDS appeared $\sim 3.5 \mathrm{~min}$ before a warning was issued. Assuming some time is needed 
to issue a warning once a TDS is observed, this may translate into a warning being issued 2-2.5 min sooner than if the TDS was not utilized. Over an average year in the United States, there are $\sim 1253$ tornadoes (NCEI 2016), of which $\sim 16 \%$ can be expected to produce a TDS (Van Den Broeke and Jauernic 2014). An average of $10 \%$ of tornadoes have negative or zero lead time (Brotzge and Erickson 2009), and $25.5 \%$ of tornadoes were unwarned before the polarimetric upgrade (Brotzge and Erickson 2010). For simplicity, it could be assumed that these rates are the same for tornadoes that produce TDSs as for those that do not. This is not quite the case, because tornadoes that are unwarned and that have negative lead times are likely weaker events and thus less likely to have a TDS. Thus, these results represent an "upper bound" on the value of the TDS for tornado warning statistics. With this "upper bound" assumption, utilization of the TDS may result in $\sim 45$ previously unwarned tornadoes being warned annually, and in $\sim 65$ additional tornadoes being warned with less negative lead time. This is thought to represent a substantial improvement in overall tornado warning statistics. Future work should examine how the TDS is utilized in the tornado warning process once the use of polarimetric radar variables has been engrained and once an automated TDS detection algorithm (Snyder and Ryzhkov 2015) becomes operational.

Acknowledgments. Nick McElhose is acknowledged for his help gathering tornado warning and update times and text. The peer reviewers are thanked for their valuable contributions to the manuscript. The author is supported by an academic appointment at the University of Nebraska-Lincoln.

\section{REFERENCES}

Bodine, D. J., M. R. Kumjian, R. D. Palmer, P. L. Heinselman, and A. V. Ryzhkov, 2013: Tornado damage estimation using polarimetric radar. Wea. Forecasting, 28, 139-158, CrossRef.

Brotzge, J., and S. Erickson, 2009: NWS tornado warnings with zero or negative lead times. Wea. Forecasting, 24, 140-154, CrossRef. , and _ 2010: Tornadoes without NWS warning. Wea. Forecasting, 25, 159-172, CrossRef. , and W. Donner, 2013a: The tornado warning process: A review of current research, challenges, and opportunities. Bull. Amer. Meteor. Soc., 94, 1715-1733, CrossRef.
, S. Erickson, and H. Brooks, 2011: A 5-yr climatology of tornado false alarms. Wea. Forecasting, 26, 534-544, CrossRef.

, S. E. Nelson, R. L. Thompson, and B. T. Smith, 2013b: Tornado probability of detection and lead time as a function of convective mode and environmental parameters. Wea. Forecasting, 28, 1261-1276, CrossRef.

Chaney, P., and G. Weaver, 2008: The Super Tuesday tornado disaster at Lafayette, Tennessee: Preparedness, response, and previous experience. Natural Hazards Center Quick Response Rep. 198.

Corder, G. W., and D. I. Foreman, 2014: Nonparametric Statistics: A Step-by-Step Approach. Wiley. ISBN: 9781118840313.

Fuhrmann, C. M., C. E. Konrad II, M. M. Kovach, J. T. McLeod, W. G. Schmitz, and P. G. Dixon, 2014: Ranking of tornado outbreaks across the United States and their climatological characteristics. Wea. Forecasting, 29, 684-701, CrossRef.

Hoekstra, S., K. Klockow, R. Riley, J. Brotzge, H. Brooks, and S. Erickson, 2011: A preliminary look at the social perspective of warn-on-forecast: Preferred tornado warning lead time and the general public's perceptions of weather risks. Wea. Clim. Soc., 3, 128-140, CrossRef.

Houser, J. L., H. B. Bluestein, and J. C. Snyder, 2016: A finescale radar examination of the tornadic debris signature and weak-echo reflectivity band associated with a large, violent tornado. Mon. Wea. Rev., 144, 4101-4130, CrossRef.

Jauernic, S. T., and M. S. Van Den Broeke, 2017: Tornado warning response and perceptions among undergraduates in Nebraska. Wea. Clim. Soc., 9, 125-139, CrossRef.

Jones, T. A., K. M. McGrath, and J. T. Snow, 2004: Association between NSSL mesocyclone detection algorithmdetected vortices and tornadoes. Wea. Forecasting, 19, 872-890, CrossRef.

Mileti, D. D., and J. H. Sorensen, 1990: Communication for emergency public warnings: A social science perspective and state-of-the-art assessment. Oak Ridge National Laboratory Rep. ORNL-6609, 165 pp.

Mitchell, E. D., S. V. Vasiloff, G. J. Stumpf, A. Witt, M. D. Eilts, J. T. Johnson, and K. W. Thomas, 1998: The National Severe Storms Laboratory tornado detection algorithm. Wea. Forecasting, 13, 352-366, CrossRef.

National Centers for Environmental Information, 2016: U.S. tornado climatology. [Available online at https://www. ncdc.noaa.gov/climate-information/extreme-events/ustornado-climatology]

Quoetone, E., J. Boettcher, and C. Spannagle, 2009: How did that happen? A look at factors that go into forecaster warning decisions. 34th Annual Meeting of the National Weather Association, Norfolk, VA. National Weather Association. [Available online at www.nwas.org/ meetings/nwa2009/]. 
Ryzhkov, A. V., T. J. Schuur, D. W. Burgess, and D. S. Zrnić, 2005: Polarimetric tornado detection. J. Appl. Meteor., 44, 557-570, CrossRef

Saari, M. D. W., R. M. Lawton, C. J. Schultz, and L. D. Carey, 2014: Early characteristics of the polarimetric tornadic debris signature associated with the 20 May 2013 Newcastle-Moore, Oklahoma, tornado. J. Operational Meteor., 2(10), 110-114, CrossRef.

Schultz, C. J., and Coauthors, 2012a: Dual-polarization tornadic debris signatures part I: Examples and utility in an operational setting. Electronic J. Oper. Meteor., 13, 120-137, CrossRef.

, and , 2012b: Dual-polarization tornadic debris signatures part II: Comparisons and caveats. Electronic J. Oper. Meteor., 13, 138-150, CrossRef.

Sherman-Morris, K., 2010: Tornado warning dissemination and response at a university campus. Nat. Hazards, $\mathbf{5 2}$, 623-638, CrossRef.

Smith, B. T., R. L. Thompson, J. S. Grams, C. Broyles, and H. E. Brooks, 2012: Convective modes for significant severe thunderstorms in the contiguous United States. Part I: Storm classification and climatology. Wea. Forecasting, 27, 1114-1135, CrossRef.

, A. R. Dean, and P. T. Marsh, 2015: Diagnosing the conditional probability of tornado damage rating using environmental and radar attributes. Wea. Forecasting, 30, 914-932, CrossRef.

Snyder, J. C., and A. V. Ryzhkov, 2015: Automated detection of polarimetric tornadic debris signatures using a hydrometeor classification algorithm. J. Appl. Meteor. Climatol., 54, 1861-1870, CrossRef.

Spoden, P. J., R. A. Wolf, and L. R. Lemon, 2012: Operational uses of spectrum width. Elec. J. Severe Storms Meteor., 7(2), 1-28, CrossRef.

Van Den Broeke, M. S., 2015: Polarimetric tornadic debris signature variability and debris fallout signatures. $J$. Appl. Meteor. Climatol., 54, 2389-2405, CrossRef.

and S. T. Jauernic, 2014: Spatial and temporal characteristics of polarimetric tornadic debris signatures. J. Appl. Meteor. Climatol., 53, 2217-2231, CrossRef.

Warning Decision Training Division, cited 2016: WSR88D dual-polarization radar decision aid. National Weather Service Warning Decision Training Division. [Available online at http://www.wdtb.noaa.gov/courses/ dualpol/Outreach/DualPol-Flipchart.pdf]

Yu, T.-Y., Y. Wang, A. Shapiro, M. B. Yeary, D. S. Zrnić, and R. J. Doviak, 2007: Characterization of tornado spectral signatures using higher-order spectra. J. Atmos. Oceanic Technol., 24, 1997-2013, CrossRef. 